\title{
Facteurs non-génétiques influençant le poids et la croissance de veaux de race Borgou à la Ferme d'élevage de l'O kpara au Bénin
}

\author{
A.K.I. Youssao ${ }^{1,2 *}$ A. Ahissou ${ }^{3}$ C. Michaux ${ }^{2}$ \\ F. Farnir ${ }^{2}$ Z. Touré ${ }^{3}$ N.-D. Idrissou ${ }^{3}$ P.L. Leroy ${ }^{1,2}$
}

\begin{abstract}
Mots-clés
Bovin Borgou - Veau - Poids à la naissance - Gain de poids - Saison - Age . Mère - Sexe - Facteur de croissance Bénin.
\end{abstract}

Résumé

\begin{abstract}
L'étude de quelques facteurs non-génétiques influençant le poids à la naissance, à trois et à douze mois, ainsi que les gains quotidiens moyens de la naissance à trois mois, de la naissance à douze mois et de trois à douze mois de veaux Borgou a été réalisée à partir des données issues de 453 veaux élevés à la Ferme d'élevage de l'O kpara de 1994 à 1998. Un modèle linéaire incluant les effets fixes de la saison de naissance, de l'année de naissance, de l'âge de la mère au vêlage et du sexe a été utilisé dans le cadre de l'analyse de variance par les moindres carrés. La saison de naissance a eu un effet significatif ( $p<0,001$ ) sur les poids et les gains quotidiens moyens. Les meilleures performances pondérales ont été obtenues sur des animaux nés pendant la saison pluvieuse. L'année de naissance a influencé significativement $(p<0,001)$ le poids à la naissance, à douze mois et le gain quotidien moyen de la naissance à douze mois. L'effet de l'âge de la mère au vêlage a été observé chez les veaux âgés de douze mois $(p<0,05)$ et sur le gain quotidien moyen enregistré de la naissance à douze mois $(p<0,05)$. Les mâles avaient un poids significativement supérieur à celui des femelles à la naissance $(p<0,05)$.
\end{abstract}

\section{INTRO DUCTIO N}

La race Borgou est issue d'un croisement lointain stabilisé entre les taurins à courtes cornes (Somba et, accessoirement, Lagunaire) et les zébus, principalement le White Fulani (6). Originaire du département du Borgou au Bénin, son aire de distribution géographique s'étend au Nigeria (Keteku), au Burkina Faso (Méré) et au Togo (7). Au Bénin, cette race représente 34 p. 100 de l'effectif national bovin (8) et son mode d'élevage est de type extensif et traditionnel (sédentaire ou transhumant), basé sur l'exploitation du pâturage naturel (4). Cette race possède une trypanotolérance au même titre que les races des Lagunes ou N'Dama (5). L'amélioration génétique de la race Borgou a été entreprise à la Ferme d'élevage de l'Okpara en 1952

\footnotetext{
1. Université de Liège, Institut vétérinaire tropical - bât. 43, 20 boulevard de Colonster, 4000 Liège, Belgique

2. Université de Liège, Faculté de Médecine vétérinaire, département de Génétique bât. 43, 20 boulevard de Colonster, 4000 Liège, Belgique

3. Direction de l'élevage, Projet pour le développement de l'élevage, Ferme d'élevage de l'Okpara, BP 33, Parakou, Bénin

* Auteur pour la correspondance

Tél : +32 (0)4 3664150 ; fax : +32 (0)4 3664122

E-mail : iyoussao@student.ulg.ac.be
}

par croisement avec la race N'Dama importée du Sénégal, de la Côte d'Ivoire et de la Guinée (17). Cet essai d'amélioration s'est soldé par un échec attribuable au manque de suivi après la phase du projet. Dans le cadre du Projet pour le développement de la production animale (Pdpa) - l'actuel Projet pour le développement de l'élevage au Bénin —, la Ferme de l'Okpara a repris l'amélioration des performances zootechniques de la race Borgou. Depuis octobre 1994, cette amélioration se fait en race pure en se basant sur un programme de sélection massale faisant intervenir le poids, la robe et la conformation phénotypique. Les performances de production et de reproduction de la race Borgou varient selon que le mode d'élevage est traditionnel (sédentaire ou transhumant), semi-amélioré ou amélioré $(3,4,9,10,11,12)$. L'objectif de ce travail a été d'étudier l'influence des principaux facteurs non-génétiques et, plus particulièrement, leur importance relative sur les caractères de croissance des veaux nés et élevés selon le mode semi-amélioré de 1994 à 1998 à la Ferme de l'Okpara.

\section{MATERIEL ET METHODES}

Les données de poids à la naissance, à trois et à douze mois, puis celles du gain quotidien moyen de la naissance à trois mois, de trois à douze mois et de la naissance à douze mois ont été étudiées 
sur 453 veaux de race Borgou de 1994 à 1998, l'effectif annuel moyen des bovins Borgou ayant été de 2577 têtes. Les paramètres pris en compte dans le cadre de cette étude ont été la saison de naissance, l'année de naissance, l'âge de la mère au vêlage et le sexe.

\section{Milieu de l'étude}

La Ferme de l'Okpara s'étend sur 33000 hectares dont 5000 à peine sont exploités. Elle est située dans la sous-préfecture de Kika, département du Borgou au Bénin, à $15 \mathrm{~km}$ à l'est de la ville de Parakou. Le climat est de type soudanien avec en alternance une saison pluvieuse (mai à octobre) et une saison sèche (novembre à avril) marquée par l'Harmattan (décembre à février). La pluviométrie est de $1200 \mathrm{~mm}$ environ et la température annuelle moyenne varie entre 26 et $27^{\circ} \mathrm{C}$. Les températures les plus élevées sont enregistrées en mars et en avril, les plus basses en décembre et en janvier. Le relief est constitué d'une pénéplaine cristalline comportant des collines à roches dures. De grandes dépressions permettent l'écoulement des eaux de pluie vers le fleuve Okpara et son affluent la Dama, principales sources d'abreuvement pour le bétail. Le sol, de texture sableuse, sabloargileuse ou limoneuse par endroits, supporte une végétation de savane. Celle-ci est affectée chaque année par les feux de brousse.

\section{Mode d'élevage}

Le mode d'élevage était de type semi-amélioré et les troupeaux étaient constitués selon le sexe et l'âge des animaux. En décembre 1997, la Ferme de l'Okpara comptait 2484 bovins Borgou répartis en 13 troupeaux dont 1 pour les taureaux, 2 pour les taurillons, 6 pour les vaches et les veaux, 2 pour les génisses 1 (1 à 2 ans) et 2 pour les génisses 2 ( 2 à 3 ans). Ces animaux passaient la journée au pâturage et la nuit dans un parc muni d'abreuvoirs et de mangeoires. En général, dans le département du Borgou, l'alimentation des bovins était basée sur l'exploitation du pâturage naturel.

La composition et l'évolution de la valeur nutritive des pâturages naturels varient en fonction de la pluviosité (20). Pendant la saison pluvieuse, de mai à octobre, les parcours naturels repoussent et le pâturage est abondant. Les graminées dominent dans la strate herbacée dont les espèces sont consommées à des stades de développement et à des degrés divers. Les genres de graminées les plus consommés sont Andropogon, Hyparrhenia, Pennisetum, Setaria. La composition chimique et la valeur énergétique de ces graminées varient aussi en fonction des saisons et des espèces (20). Les graminées sont préférées pendant cette période et constituent par conséquent la majeure partie de la phytomasse ingérée. La fin de la période de croissance (novembre-décembre) coïncide souvent avec l'avènement des feux de brousse annuels. Les parcours naturels restent nus pendant les quatre premiers mois de l'année et le pâturage est essentiellement à base de fourrages ligneux.

Pendant cette période, seuls les bas-fonds et les abords des cours d'eaux de l'Okpara et de ses affluents offraient des graminées vertes, alors très peu abondantes à la Ferme de l'Okpara. Les animaux ont également bénéficié des résidus de récoltes issus des champs de cultures. Les prairies artificielles étaient constituées de Brachiaria ruziziensis (33 ha en 1995), de Stylosanthes sp. (15 ha en 1996) et d'Andropogon gayanus (2 ha en 1995). Brachiaria ruziziensis était utilisé sous forme d'ensilage ou de foin alors que Stylosanthes sp. l'était surtout pour l'ensilage. Les exploitations sur pied des prairies artificielles se faisaient surtout sur les anciennes parcelles. Une complémentation en ensilage, foin, graines de coton, sel de cuisine et pierre à lécher était assurée aux animaux de la ferme en fonction des disponibilités pendant la période de transition entre la saison sèche et la saison des pluies (janvier à avril).

Le suivi sanitaire était basé sur l'administration de vitamines et d'oligo-éléments, des traitements préventifs contre la trypanosomose, les parasitoses gastro-intestinales, les tiques et autres arthropodes. Le programme national de prophylaxie contre les grandes épizooties (pasteurellose, peste bovine, péripneumonie contagieuse bovine, charbon bactéridien) était suivi régulièrement. Des traitements spécifiques étaient pratiqués pour les maladies occasionnelles en fonction des cas cliniques.

Un programme de regroupement des naissances à été conçu et mis en application en octobre 1994, mettant fin à la monte libre. En 1995, deux montes ont été organisées, l'une en janvier-février et l'autre d'août à octobre. Les naissances ont ainsi été enregistrées respectivement en octobre-novembre 1995 et en juin-juillet 1996. Quant aux années 1996 et 1997, les montes ont eu lieu en janvierfévrier et en août-septembre et les naissances respectivement en octobre-novembre et en mai-juin.

Un programme de sélection massale basé sur les performances individuelles en vue d'uniformiser la race Borgou a été mis en place à partir de 1994 à la Ferme de l'Okpara. Ce programme porte sur les veaux au sevrage, les génisses, les vaches et les taureaux. Les veaux dont le poids au sevrage est supérieur à $100 \mathrm{~kg}$ sont présélectionnés pour la catégorie des taurillons âgés de 12 à 24 mois (type 1). Les taurillons de type 1 dont le poids est supérieur à $200 \mathrm{~kg}$ entre 18 et 24 mois sont retenus pour la catégorie des taurillons âgés de 2 à 3 ans (type 2). Les animaux non retenus sont castrés et destinés à la boucherie, à la culture attelée ou à d'autres fins. Les taurillons de type 2 qui présentent une bonne conformation et la meilleure performance pondérale sont sélectionnés pour être géniteurs. Le poids minimal au sevrage pour les veaux femelles est de $100 \mathrm{~kg}$ et pour les génisses de type 1 (12 à 24 mois) de $120 \mathrm{~kg}$. Les critères phénotypiques sont également pris en compte lors de la sélection : robe blanche, mufle, cornes et pis noirs, fanon et bosse peu développés. Ce schéma de sélection concerne tous les bovins Borgou de la ferme.

Les animaux étaient pesés sur une bascule pèse bétail. Une fiche de suivi a été établie et comprenait le numéro du parc, le numéro du veau, le numéro de la mère, la saison de naissance, l'année de naissance, l'âge de la mère au vêlage, le sexe, le poids à la naissance $(\mathrm{P} 0)$, le poids à trois mois $(\mathrm{P} 3)$, le poids à douze mois $(\mathrm{P} 12)$, le gain quotidien moyen entre la naissance et trois mois (Gqm03), le gain quotidien moyen entre trois et douze mois (Gqm312), ainsi que le gain quotidien moyen entre la naissance et douze mois (Gqm012). A la naissance, tous les veaux de la ferme étaient pesés, puis, en fonction de la charge de travail du personnel, un échantillon de taille variable (10 à 20 veaux) était choisi sur la base de la conformation phénotypique de la race Borgou (robe blanche, mufle, cornes et pis noirs) et pesé mensuellement.

\section{Analyse statistique}

Le modèle linéaire fixe suivant a été ajusté aux données de poids et de croissance :

$$
\mathrm{Y}_{\mathrm{ijklm}}=\mu+\mathrm{S}_{\mathrm{i}}+\mathrm{AN}_{\mathrm{j}}+\mathrm{AV}_{\mathrm{k}}+\mathrm{S}_{1}+\mathrm{E}_{\mathrm{ijklm}}
$$

$\mathrm{Y}_{\mathrm{ijklm}}$ est un poids ou un gain du $\mathrm{m}^{\mathrm{e}}$ veau, né dans la saison $\mathrm{i}$, l'année j, à l'âge au vêlage de la mère $\mathrm{k}$ et au sexe 1 ;

$\mu$ est la valeur de la moyenne générale ;

$\mathrm{S}_{\mathrm{i}}$ est l'effet fixe de la saison de vêlage i. Il y a quatre saisons : S1, de décembre à février (saison sèche); S2, de mars à mai (transition 
entre la saison sèche et la saison des pluies) ; S3, de juin à août (saison des pluies); $\mathrm{S} 4$, de septembre à novembre (transition entre la saison des pluies et la saison sèche);

$\mathrm{AN}_{\mathrm{j}}$ est l'effet fixe de l'année de naissance j (1994 à 1997) (les veaux nés en 1997 n'avaient pas encore atteint 12 mois lors de l'analyse des données) ;

$\mathrm{AV}_{1}$ est l'effet fixe de l'âge de la mère $\mathrm{k}$ au vêlage ( 5 classes : 3 et 4, 5, 6, 7, 8 à 13 ans) ;

$\mathrm{S}_{\mathrm{m}}$ est l'effet fixe du sexe 1 (2 classes : mâle et femelle);

$\mathrm{E}_{\mathrm{ijklm}}$ est l'effet résiduel aléatoire.

Les interactions entre ces facteurs de variation ne sont pas significatives et, par conséquent, n'ont pas été prises en compte dans le modèle ci-dessus. Les données ont été analysées selon la procédure GLM de SAS (18), puis les moyennes des moindres carrés ont été estimées et comparées par le test t de Student. La part de la variation expliquée par le modèle et chacun des facteurs non-génétiques a été calculée selon la méthodologie décrite par Searle (19), en utilisant la différence de la part de variation $\mathrm{R}^{2}$ (réduction de la somme des carrés suite à la présence ou à l'absence du facteur).

\section{RESU LTATS}

L'effectif, la moyenne, l'écart-type, le minimum et le maximum des performances pondérales sont présentés au tableau I pour l'ensemble des veaux, par sexe et par année de naissance. Les résultats de l'analyse de variance montrent la signification de chaque facteur de variation inclus dans le modèle d'analyse (tableau II). La part de la variation totale $\left(\mathrm{R}^{2}\right)$ des poids et des gains quotidiens moyens expliquée par chacun des facteurs nongénétiques est présentée à la figure 1 . L'importance de la variation expliquée par le modèle linéaire global a été de 18,9, 22,5 et 27,2 p. 100, respectivement pour le poids à la naissance, à trois mois et pour le gain quotidien moyen de la naissance à trois mois. Elle a été supérieure à $30 \mathrm{p} .100$ pour le poids à douze mois et pour Gqm012 et Gqm312. La part de la variation totale due à la saison de naissance a été la plus importante de tous les effets du modèle et a varié de 6,5 p. 100 pour le poids à la naissance à 19,1 p. 100 pour Gqm312. Le deuxième, en importance, effet du modèle a été l'année de naissance ; la part de la variation totale a surtout été observée à douze mois (19,1 p. 100). Elle a été de 5,3 p. 100 pour le poids à la naissance, et respectivement de 10,3 et 10,5 p. 100 pour Gqm012 et Gqm312. Quant à l'âge de la mère au vêlage, la part de variation la plus significative a été obtenue pour le poids des veaux âgés de douze mois $(4,8$ p. 100). La contribution du sexe à la variation totale a été plus élevée à la naissance $(4,7$ p. 100), pour Gqm012 (2,1 p. 100) et Gqm312 (5 p. 100). Comme certaines performances ne pouvaient être disponibles lors de la collecte des données (tableau I), les degrés de liberté relatifs à l'erreur résiduelle ont varié de 226 à 339 pour les différents paramètres étudiés (tableau II). Les moyennes des moindres carrés sont indiquées dans les tableaux III et IV.

La saison de vêlage a été l'effet significatif le plus important observé sur les poids et les gains quotidiens moyens $(\mathrm{p}<0,001)$. Les veaux nés vers la fin de la saison des pluies et au début de la saison sèche $(\mathrm{S} 4)$ ont été plus lourds à la naissance $(\mathrm{p}<0,05)$ que ceux nés aux autres saisons. A trois mois, le poids moyen des veaux nés pendant la saison de transition (S4) et la saison sèche (S1) a été inférieur à $40 \mathrm{~kg}$. Ceux qui sont nés pendant la dernière phase de la saison sèche et en saison pluvieuse (S2 et S3) ont été les plus lourds avec un poids moyen supérieur à $43 \mathrm{~kg}$. Les veaux nés en saison de transition (S2) et en saison pluvieuse ont présenté les meilleurs gains quotidiens moyens de la naissance à trois mois. Les veaux nés pendant l'hivernage et la saison de transition (S4) ont eu un poids moyen supérieur à $100 \mathrm{~kg}$ à 12 mois, avec une meilleure croissance pondérale de trois à douze mois. Une différence significative $(\mathrm{p}<0,05)$ d'une saison à l'autre a marqué les gains quotidiens moyens de la naissance à douze mois.

L'année de naissance a eu un effet significatif important ( $\mathrm{p}<0,001)$ sur le poids à la naissance, à douze mois, ainsi que pour Gqm312 et Gqm012. Les poids à la naissance, à 12 mois, Gqm312 et Gqm012 ont été nettement inférieurs pour les veaux nés en 1996 (tableaux III et IV).

L'âge de la mère au vêlage a eu un effet significatif $(p<0,05)$ sur le poids du veau à 12 mois. Le poids à 12 mois a augmenté en fonction de l'âge de la mère au vêlage de trois à sept ans et a chuté à partir de la $8^{\mathrm{e}}$ année. Le poids le plus important de $112,3 \mathrm{~kg}$ $(\mathrm{p}<0,05)$ a été observé chez les veaux issus des vaches âgées de sept ans (tableau III). Le gain quotidien moyen de la naissance à douze mois le plus important a également été observé chez les veaux nés de vaches âgées de sept ans (tableau IV).

Enfin, le sexe a eu un effet très significatif $(\mathrm{p}<0,001)$ sur le poids à la naissance, ainsi que pour Gqm312 et Gqm012. Les mâles ont présenté un poids à la naissance significativement plus élevé $(\mathrm{p}<0,05)$ que les femelles. Cette différence n'a plus été observée à trois mois ni à douze. Pour le gain quotidien moyen, une différence significative entre les mâles et les femelles a été observée de la naissance à douze mois et de trois à douze mois $(\mathrm{p}<0,05)$.

\section{DISCUSSION}

Dans la zone tropicale, la disponibilité fourragère est fonction de la pluviosité. L'aliment de base étant le pâturage naturel, la production laitière des vaches allaitantes dépend des saisons. Selon Hoste et coll. (13) et Dehoux (4), la moitié de la production laitière est obtenue au cours des trois premiers mois de lactation et le lait constitue un facteur limitant de l'expression du poids et de la vitesse de croissance des veaux.

Les veaux nés en pleine saison sèche $(\mathrm{S} 1)$ ont été âgés de trois mois pendant la saison de transition $\mathrm{S} 2$ et de douze mois en pleine saison sèche. De ce fait, les différents poids et gains de poids se sont trouvés défavorisés.

Les veaux nés dans la dernière phase de la saison sèche et au début de la saison pluvieuse (S2) ont atteint à l'âge de trois mois les poids les plus importants $(48,1 \mathrm{~kg})$. Le gain quotidien moyen de la naissance à trois mois a également été le plus élevé. Ces veaux ont traversé toute la saison sèche jusqu'à l'âge de 12 mois, avec pour conséquence le poids le plus faible à 12 mois.

Les veaux nés en pleine saison des pluies (S3) ont présenté les poids les plus élevés à 12 mois. Les mois de septembre et d'octobre correspondent à la période d'épiaison des pâturages et à la fin de la saison pluvieuse (20). A partir de novembre, le pâturage se transforme en pailles qui sont ensuite brûlées par les feux de brousse. A cette époque, les vaches ont bénéficié d'apport des résidus de récolte et des compléments alimentaires qui ont contribué au maintien du poids des veaux à trois mois $(43,3 \mathrm{~kg})$ et du gain quotidien moyen de la naissance à trois mois $(278 \mathrm{~g})$. Par ailleurs, après l'âge de trois mois, les animaux avaient déjà traversé la quasi-totalité de la saison pluvieuse où le pâturage était abondant et de qualité optimale. Les gains quotidiens moyens (Gqm012 et Gqm312) ont ainsi été plus importants que ceux enregistrés chez les veaux nés pendant les autres saisons. 
Tableau I

Effectif, moyenne, écart-type, minimum et maximum des performances pondérales de veaux Borgou à la Ferme de l'O kpara

\begin{tabular}{|c|c|c|c|c|c|c|}
\hline & Performance & Effectif * & Moyenne & Ecart-type & Minimum & Maximum \\
\hline \multirow{6}{*}{$\begin{array}{l}\text { Moyenne générale } \\
\text { (453) ** }\end{array}$} & $\mathrm{PO}(\mathrm{kg})$ & 451 & 18,86 & 1,87 & 13 & 25 \\
\hline & P3 (kg) & 440 & 41,12 & 8,6 & 23 & 66 \\
\hline & $\mathrm{P} 12$ (kg) & 391 & 102,21 & 23,41 & 56 & 176 \\
\hline & Gqm03 (g) & 438 & 246 & 0,9 & 111 & 522 \\
\hline & Gqm312 (g) & 391 & 174 & 1,49 & 122 & 444 \\
\hline & Gqm012 (g) & 390 & 190 & 1,11 & 118 & 414 \\
\hline
\end{tabular}

Sexe

(221)

$\mathrm{PO}(\mathrm{kg}) \quad 221$

221

$\begin{array}{ll}\text { P3 }(\mathrm{kg}) & 216 \\ \text { P12 }(\mathrm{kg}) & 188\end{array}$

P12 (kg)

188

$\mathrm{Gqm03}(\mathrm{g})$

216

Gqm312 (g)

185

Gqm012 (g)

180

Mâle

PO $(\mathrm{kg})$

P3 $(\mathrm{kg})$

230

(230)

P12 $(\mathrm{kg})$

Gqm03 (g)

224

203

Gqm312 (g)

222

Gqm012 (g)

202

201

18,48
41,31
99,22
254
160
181
19,23
40,92
104,96
239
189
199

1,65

7,9

23,56

0,86

1,48

1,12

1,99

9,25

22,98

0,9

1,49

1,1
15,5

24

56

95

150

111

13

23

58

111

130

123
23

62

149

478

359

346

25

66

176

522

444

413

\section{Année de naissance}

1994

(99)

1995

(234)

1996

(89)

Pour les veaux nés à la fin de la saison des pluies et au début de la saison sèche (S4), malgré la complémentation alimentaire des vaches, le poids moyen à trois mois n'était pas différent de celui observé chez les veaux nés en saison sèche. Après cet âge, les animaux ont bénéficié de l'abondance des fourrages pendant toute la saison pluvieuse avec les gains quotidiens moyens les plus importants de la naissance à douze mois et de trois à douze mois.

Les résultats de cette étude confirment que le regroupement des naissances en juin-juillet et en octobre-novembre organisé à la de trois à douze mois ; Gqm012: gain quotidien moyen de la naissance à douze mois

$\begin{array}{ccccc}99 & 19,21 & 2,15 & 13 & 25 \\ 99 & 37,79 & 7,75 & 25 & 56 \\ 99 & 112,16 & 26,11 & 56 & 176 \\ 99 & 206 & 0,8 & 133 & 355 \\ 99 & 250 & 1,28 & 115 & 444 \\ 99 & 236 & 0,98 & 118 & 414 \\ 234 & 19,23 & 1,81 & 15,5 & 25 \\ 234 & 42,1 & 8,91 & 24 & 66 \\ 212 & 103,63 & 17,41 & 57 & 147 \\ 234 & 254 & 0,93 & 95 & 522 \\ 212 & 192 & 1,25 & 122 & 348 \\ 212 & 205 & 0,94 & 91 & 342 \\ 89 & 17,91 & 1,28 & 15,5 & 21,5 \\ 78 & 44,61 & 7,17 & 30 & 60 \\ 86 & 87,93 & 26,55 & 58 & 133 \\ 76 & 295 & 0,76 & 161 & 461 \\ 77 & 139 & 1,18 & 104 & 270 \\ 80 & 179 & 0,85 & 116 & 312 \\ 29 & 17,63 & 17,63 & 16 & 20 \\ 29 & 35,24 & 35,24 & 23 & 45 \\ 29 & 195 & 1,96 & 78 & 294\end{array}$

$\mathrm{P} 0$ : poids à la naissance ; P3 : poids à trois mois ; P12 : poids à 12 mois ; Gqm03 : gain quotidien moyen de la naissance à trois mois ; Gqm312 : gain quotidien moyen

* Effectif des veaux par performance ; ** Nombre d'observations ; *** Les veaux nés en 1997 n'avaient pas atteint l'âge de 12 mois lors de la collecte des données

Ferme de l'Okpara permet d'obtenir de meilleures performances pour les veaux. L'étude des paramètres de reproduction et de production des bovins Borgou et Lagunaire réalisée par le Centre d'insémination artificielle et le Contrôle sanitaire des reproducteurs (3) a montré l'existence de l'effet saison de naissance sur le poids et la croissance des veaux. Dans ce centre, le poids à la naissance des veaux Borgou était de $16,4 \mathrm{~kg}$ en petite saison des pluies, de $16 \mathrm{~kg}$ en grande saison des pluies et de $15 \mathrm{~kg}$ en saison sèche. Ces résultats confirment ceux de Dehoux (4) qui a observé 
Tableau II

Résultats des analyses de la variance des poids et des gains quotidiens moyens du veau Borgou

\begin{tabular}{|c|c|c|c|c|c|c|c|c|c|c|c|c|}
\hline \multirow[t]{2}{*}{ Sources de variation } & \multicolumn{2}{|c|}{ PO } & \multicolumn{2}{|r|}{ P3 } & \multicolumn{2}{|r|}{ P12 } & \multicolumn{2}{|c|}{ G qm03 } & \multicolumn{2}{|c|}{ G qm312 } & \multicolumn{2}{|c|}{ Gqm012 } \\
\hline & ddl & SCE & ddl & SCE & ddl & SCE & ddl & SCE & ddl & SCE & ddl & SCE \\
\hline Saison de naissance & 3 & $65,21 * *$ & 3 & $2576,14 * *$ & 3 & $15103,11^{* *}$ & 3 & $0,33^{* *}$ & 3 & $0,79 * *$ & 3 & $0,38 * *$ \\
\hline Année de naissance & 3 & $53,69 * *$ & 3 & 121,21 & 2 & $23101,94^{* *}$ & 3 & 0,02 & 3 & $0,42^{* *}$ & 3 & $0,24 * *$ \\
\hline Age au vêlage (ans) & 4 & 6,22 & 4 & 344,19 & 4 & $5759,21^{*}$ & 4 & 0,04 & 4 & 0,08 & 4 & 0,05 \\
\hline Sexe & 1 & $47,21^{* *}$ & 1 & 8,71 & 1 & 833,29 & 1 & 0,01 & 1 & $0,21 * *$ & 1 & $0,11^{* *}$ \\
\hline Erreur résiduelle & 242 & 814,92 & 241 & 15602,14 & 226 & 75420,31 & 339 & 1,51 & 241 & 2,59 & 242 & 1,56 \\
\hline
\end{tabular}

$\mathrm{P} 0$ : poids à la naissance ; P3 : poids à trois mois ; P12 : poids à 12 mois ; Gqm03 : gain quotidien moyen de la naissance à trois mois ; Gqm312 : gain quotidien moyen de trois à douze mois ; Gqm012: gain quotidien moyen de la naissance à douze mois ; ddl : degré de liberté ; SCE : somme des carrés des écarts

* Significatif $(\mathrm{p}<0,05) ; * *$ Significatif $(\mathrm{p}<0,001)$

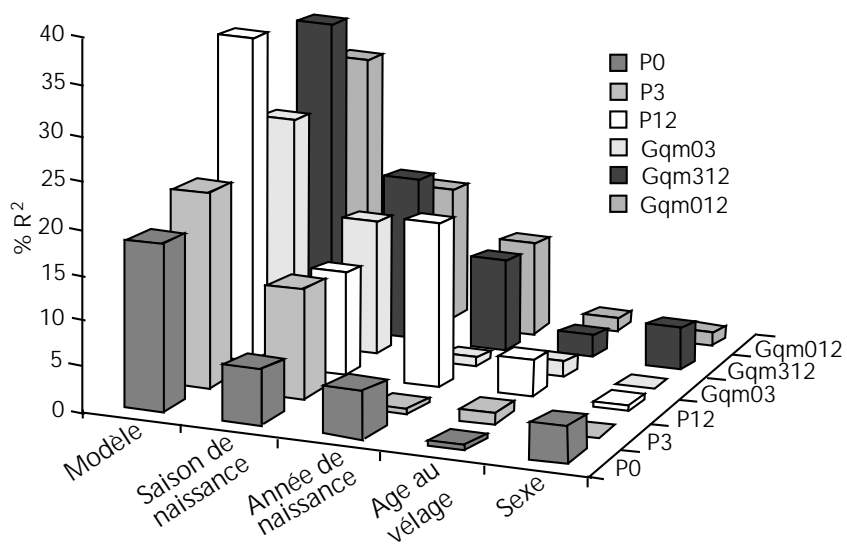

Figure 1 : part de la variation $\left(R^{2}\right)$ des poids et des gains quotidiens moyens expliquée par le modèle et les différents facteurs non-génétiques.

P0 : poids à la naissance ; $\mathrm{P3}$ : poids à trois mois ; $\mathrm{P} 12$ : poids à 12 mois. Gqm03 : gain quotidien moyen de la naissance à trois mois ; Gqm312 : gain quotidien moyen de trois à douze mois ; Gqm012 : gain quotidien moyen de la naissance à douze mois.

que les veaux nés au quatrième trimestre avaient une croissance plus importante entre le $4^{\mathrm{e}}$ et le $6^{\mathrm{e}}$ mois d'âge $(300 \mathrm{~g} / \mathrm{j})$ que ceux nés pendant les premiers mois $(248 \mathrm{~g} / \mathrm{j})$ de l'année. Poivey et coll. (16) et Planchenault et coll. (15) ont également montré que l'effet de l'année-saison était important sur le poids et la croissance des veaux et des jeunes bovins chez les races voisines Baoulé et N'Dama. Enfin, l'évolution pondérale des veaux Azawak est très peu influencée par la saison à la station de Loumbila au Burkina Faso en raison de la disponibilité fourragère et des compléments alimentaires (1).

Des variations annuelles de la croissance dues à des différences de pluviométrie ont été observées au Burkina Faso (1) et dans le nord du Cameroun (14). De 1994 à 1997, la pluviométrie et la distribution des pluies sont restées les mêmes $(9,10,11,12)$ conduisant à une certaine homogénéité des conditions climatiques. Les poids inférieurs à la naissance observés en 1996 et 1997 peuvent être expliqués par l'épidémie de fièvre aphteuse en 1996, qui a eu pour conséquence un taux de morbidité chez les veaux de 25,7 p. 100 (11), et par les épidémies de fièvre aphteuse, brucellose et dermatose nodulaire contagieuse bovine observées en 1997, où le taux de morbidité a atteint 60,9 p. 100 (12).
Pour les veaux issus des vaches âgées de trois à sept ans, le poids à douze mois et Gqm012 ont augmenté ; cette observation pourrait résulter de l'augmentation de la production laitière en fonction de l'âge de la vache. Une relation étroite a été observée entre l'effet du rang de vêlage et celui de l'âge de la mère au vêlage sur le poids et la croissance des veaux Borgou (21). Dehoux (4) a observé une augmentation de la production laitière chez la race Borgou du $1^{\text {er }}$ au $3^{\text {e }}$ rang de vêlage. Une augmentation de 34 p. 100 de la production laitière a été aussi observée chez la race N'Dama entre le $1^{\text {er }}$ et le $7^{\text {e }}$ rang de vêlage, et 72 p. 100 des variations du poids au sevrage peuvent être expliquées par la production du lait des trois premiers mois (13). La chute du poids observée après sept ans d'âge pourrait être due à l'épuisement des femelles et à la vieillesse. A la station de Loumbila au Burkina Faso, l'accroissement moyen de la production journalière de lait par rapport à la première lactation est respectivement de 0,83 , 0,34 et $0,80 \mathrm{l} / \mathrm{j}$ pour les $2^{\mathrm{e}}, 3^{\mathrm{e}}$ et $4^{\mathrm{e}}$ rangs de lactation, suivi d'une diminution respectivement de 0,39 et $0,45 \mathrm{l} / \mathrm{j}$ pour les $5^{\mathrm{e}}$ et $6^{\mathrm{e}}$ rangs de lactation (2). En milieu paysan au nord du Cameroun, Njoya et coll. (14) ont étudié le poids à la naissance des veaux suivant une régression quadratique sur le rang de vêlage ; un poids de $21,1 \mathrm{~kg}$ a été noté chez les primipares contre $24,7 \mathrm{~kg}$ au $5^{\mathrm{e}}$ vêlage.

Les résultats de cette étude sur l'effet du sexe confirment ceux du Centre d'insémination artificielle et du Contrôle sanitaire des reproducteurs (3) qui ont rapporté que les veaux mâles Borgou pesaient $16,69 \mathrm{~kg}$ à la naissance, soit $1,65 \mathrm{~kg}$ de plus que les veaux femelles. Selon le Centre, l'effet du sexe sur le gain quotidien moyen de la naissance à six mois n'est pas significatif chez la race Borgou. Dans les résultats présentés ici, une différence significative du gain quotidien moyen existait entre 3 et 12 mois et entre la naissance et 12 mois $(\mathrm{p}<0,05)$. Au ranch de MadinaDiassa au Mali, Planchenault et coll. (15) ont observé que les mâles avaient un poids et un gain significativement supérieurs à ceux des femelles chez la race N'Dama. Une différence significative a été observée à la naissance $(\mathrm{p}<0,05)$ entre le poids des mâles $(23,28 \pm 3,04 \mathrm{~kg})$ et celui des femelles $(21,50 \pm 3,23 \mathrm{~kg})$ chez le zébu Azawak (1). Les résultats de l'analyse de variance obtenus par Poivey et coll. (16) montrent que le sexe a un effet significatif $(\mathrm{p}<0,01)$ sur le poids à un âge donné et un effet nonsignificatif sur les vitesses de croissance mensuelles chez les races taurines ivoiriennes (Baoulé, N'Dama et produits croisés). 


\section{Tableau III}

Moyennes des moindres carrés et écarts-types (ET) pour les poids à la naissance,

à trois et à douze mois selon les facteurs de variation

\begin{tabular}{|c|c|c|c|c|c|c|c|c|c|}
\hline \multirow[t]{2}{*}{ Source de variation } & \multicolumn{3}{|c|}{ Poids à la naissance } & \multicolumn{3}{|c|}{ Poids à trois mois } & \multicolumn{3}{|c|}{ Poids à douze mois } \\
\hline & Effectif & $\begin{array}{l}\text { Moyenne } \\
(\mathrm{kg})\end{array}$ & ET & Effectif & $\begin{array}{l}\text { Moyenne } \\
\text { (kg) }\end{array}$ & ET & Effectif & $\begin{array}{l}\text { Moyenne } \\
\text { (kg) }\end{array}$ & ET \\
\hline \multicolumn{10}{|l|}{ Saison de naissance } \\
\hline S1 & 68 & $17,43^{a}$ & 0,56 & 68 & $37,75^{a}$ & 2,49 & 63 & $93,32^{a}$ & 3,51 \\
\hline $\mathrm{S} 2$ & 116 & $18,17^{a}$ & 0,53 & 116 & $48,09^{b}$ & 2,34 & 102 & $89,89^{a}$ & 2,77 \\
\hline S3 & 107 & $17,94^{\mathrm{a}}$ & 0,51 & 109 & $43,32^{c}$ & 2,26 & 106 & $111,72^{b}$ & 3,01 \\
\hline S4 & 160 & $18,89^{b}$ & 0,52 & 147 & $39,34^{a}$ & 2,35 & 133 & $104,26^{b}$ & 2,43 \\
\hline \multicolumn{10}{|l|}{ Année de naissance } \\
\hline 1994 & 99 & $18,89^{a}$ & 0,28 & 99 & 42,88 & 1,24 & 93 & $117,02^{a}$ & 2,95 \\
\hline 1995 & 234 & $19,13^{a}$ & 0,17 & 234 & 40,89 & 0,77 & 212 & $106,96^{b}$ & 1,87 \\
\hline 1996 & 89 & $17,49^{b}$ & 0,38 & 78 & 41,59 & 1,79 & 86 & $75,42^{c}$ & 3,79 \\
\hline 1997 & 29 & $16,92^{\mathrm{ab}}$ & 1,86 & 29 & 43,15 & 8,19 & & & \\
\hline \multicolumn{10}{|l|}{ Age au vêlage (ans) } \\
\hline 3 et 4 & 67 & 17,96 & 0,57 & 66 & 41,76 & 2,51 & 52 & $94,12^{a}$ & 3,39 \\
\hline 5 & 132 & 18,29 & 0,49 & 132 & 40,98 & 2,19 & 118 & $94,88^{a}$ & 2,44 \\
\hline 6 & 120 & 17,92 & 0,53 & 114 & 42,98 & 2,32 & 111 & $98,84^{a}$ & 2,72 \\
\hline 7 & 54 & 18,27 & 0,61 & 50 & 44,46 & 2,63 & 49 & $112,33^{b}$ & 3,67 \\
\hline 8 à 13 & 74 & 18,09 & 0,56 & 74 & 40,45 & 2,45 & 61 & $98,82^{a}$ & 3,38 \\
\hline \multicolumn{10}{|l|}{ Sexe } \\
\hline Femelle & 221 & $17,67^{a}$ & 0,49 & 216 & 41,93 & 2,16 & 188 & 97,87 & 2,1 \\
\hline
\end{tabular}

a, b, c : les moyennes avec la même lettre ne sont pas significativement différentes au seuil de 5 p. 100

$\mathrm{S} 1$ : décembre à février (saison sèche); $\mathrm{S} 2$ : mars à mai (transition entre la saison sèche et la saison des pluies) ;

$\mathrm{S} 3$ : juin à août (saison des pluies); $\mathrm{S} 4$ : septembre à novembre (transition entre la saison des pluies et la saison sèche)

Tableau IV

Moyennes des moindres carrés et écarts-types (ET) pour les gains quotidiens moyens selon les facteurs de variation

\begin{tabular}{|c|c|c|c|c|c|c|c|c|c|}
\hline \multirow[t]{2}{*}{ Source de variation } & \multicolumn{3}{|c|}{ Gqm03 } & \multicolumn{3}{|c|}{ Gqm312 } & \multicolumn{3}{|c|}{ Gqm012 } \\
\hline & Effectif & $\begin{array}{c}\text { Moyenne } \\
(\mathbf{g} / \mathbf{j})\end{array}$ & ET & Effectif & $\begin{array}{c}\text { Moyenne } \\
(\mathbf{g} / \mathrm{j})\end{array}$ & ET & Effectif & $\begin{array}{c}\text { Moyenne } \\
(\mathrm{g} / \mathrm{j})\end{array}$ & ET \\
\hline \multicolumn{10}{|l|}{ Saison de naissance } \\
\hline S1 & 68 & $224,17^{a}$ & 0,24 & 63 & $12,36^{\mathrm{a}}$ & 0,32 & 63 & $64,89^{a}$ & 0,24 \\
\hline S2 & 116 & $332,52^{b}$ & 0,23 & 102 & $40,78^{a}$ & 0,3 & 102 & $112,32^{b}$ & 0,23 \\
\hline S3 & 109 & $278,01^{c}$ & 0,22 & 106 & $161,95^{b}$ & 0,29 & 106 & $188,48^{c}$ & 0,22 \\
\hline S4 & 147 & $226,05^{a}$ & 0,23 & 133 & $131,71^{b}$ & 0,3 & 133 & $153,81^{d}$ & 0,23 \\
\hline \multicolumn{10}{|l|}{ Année de naissance } \\
\hline 1994 & 99 & 265,36 & 0,12 & 93 & $254,32^{a}$ & 0,16 & 93 & $253,19^{a}$ & 0,12 \\
\hline 1995 & 234 & 240,82 & 0,07 & 212 & $204,67^{b}$ & 0,1 & 212 & $210,76^{b}$ & 0,07 \\
\hline 1996 & 78 & 262,09 & 0,18 & 86 & $98,61^{\mathrm{c}}$ & 0,23 & 78 & $139,21^{\mathrm{C}}$ & 0,16 \\
\hline 1997 & 29 & 292,48 & 0,81 & & & & & & \\
\hline \multicolumn{10}{|l|}{ Age au vêlage (ans) } \\
\hline 3 et 4 & 67 & 264,26 & 0,25 & 52 & 52,44 & 0,32 & 52 & $104,82^{\mathrm{a}}$ & 0,24 \\
\hline 5 & 132 & 250,95 & 0,22 & 118 & 91,65 & 0,28 & 118 & $130,14^{a b}$ & 0,21 \\
\hline 6 & 120 & 277,59 & 0,23 & 111 & 73,23 & 0,29 & 111 & $123,11^{a b}$ & 0,23 \\
\hline 7 & 56 & 284,74 & 0,26 & 49 & 124,73 & 0,33 & 49 & $162,19^{b}$ & 0,26 \\
\hline 8 à 13 & 74 & 248,41 & 0,24 & 61 & 91,45 & 0,31 & 61 & $129,12^{a b}$ & 0,24 \\
\hline \multicolumn{10}{|l|}{ Sexe } \\
\hline Femelle & 221 & 268,56 & 0,21 & 216 & $58,02^{a}$ & 0,27 & 216 & $109,44^{a}$ & 0,21 \\
\hline Mâle & 230 & 261,82 & 0,21 & 224 & $115,38^{b}$ & 0,28 & 224 & $150,31^{b}$ & 0,21 \\
\hline
\end{tabular}

a, b, c : les moyennes avec la même lettre ne sont pas significativement différentes au seuil de 5 p. 100 ;

Gqm03 : gain quotidien moyen de la naissance à trois mois; Gqm312 : gain quotidien moyen de trois à douze mois ; Gqm012 : gain quotidien moyen de la naissance à douze mois ; S1 : décembre à février (saison sèche); S2 : mars à mai (transition entre la saison sèche et la saison des pluies) ; S3 : juin à août (saison des pluies) ; S4 : septembre à novembre (transition entre la saison des pluies et la saison sèche) 


\section{CONCLUSION}

L'étude des facteurs de variation non-génétiques influençant le poids et la croissance des veaux est une étape nécessaire à l'amélioration génétique de la race Borgou. La saison de naissance a été le facteur ayant eu l'effet le plus important. Le regroupement des naissances en saison pluvieuse (S3 et S4) serait favorable à la croissance des veaux. Les variations observées en 1996 et en 1997 nécessitent le renforcement des prophylaxies sanitaire et médicale dans la perspective d'améliorer la productivité du cheptel. L'effet de l'âge de la mère au vêlage n'a été observé que sur le gain quotidien moyen de la naissance à douze mois et sur le poids à douze mois. Les effets des facteurs de variation non-génétiques mis en évidence indiquent la nécessité d'ajuster les poids et les gains des animaux pour l'amélioration de l'efficacité du programme de sélection.

\section{Remerciements}

Les auteurs remercient M. El Fadili, A. Vermeylen, G. Hounsoun-vê, Kashala, H. Boly, H. Banga M'Boko, C. Chrysostome, J.-L. Hornick, S. Berghmans, les personnels de la Ferme de l'Okpara, du Projet pour le développement de l'élevage au Bénin (Pde), du département de Génétique et de l'Institut vétérinaire tropical de la Faculté de Médecine vétérinaire de l'Université de Liège (Belgique) pour leur contribution.

\section{BIBLIO GRAPHIE}

1. BOLY H., SOME S.S., KABRE A., SAWADO GO L., LEROY P.L., 2000. Reproduction et croissance du zébu Azawak en zone soudanosahélienne (Station de Loumbila au Burkina Faso). Ann. Univ. O uagadougou, 8 (série B) : 85-98.

2. BO LY H., SOME S.S., KABRE A., SAWADO GO L., LEROY P.L., 2000. Performance laitière du zébu Azawak en zone soudano-sahélienne (Station de Loumbila au Burkina Faso). Ann. Univ. Ouagadougou, 8 (série B) : 127-139.

3. CIA-CSR, 1996. Contribution à la connaissance des paramètres de reproduction et de production des bovins de races Borgou et Lagunaire. Rapport final UNB/FSA/CIA. Abomey Calavi, Bénin, Cia-Csr, 44 p.

4. DEHOUX J.P., 1993. Productivité de la race bovine Borgou en milieu traditionnel au nord-est du Bénin. Mémoire MSc, Institut de médecine tropicale, Anvers, Belgique, 97 p. ( $\left.{ }^{\circ} 98\right)$

5. DOKO S.A., 1991. Etude de la trypanosomiase et de la trypanotolérance bovine au Bénin. Mémoire MSc, Institut de médecine tropicale, Anvers, Belgique, 86 p. $\left(\mathrm{N}^{\circ} 14\right)$

6. DOMINGO A.M., 1976. Contribution à l'étude de la population bovine des Etats du golfe du Bénin. Thèse Doct. vét., Eismv, Dakar, Sénégal, $148 \mathrm{p}$.

7. FAO-PNUD, 1989. In : Deux ateliers de travail sur la reproduction du bétail trypanotolérant en Afrique occidentale et centrale, Projet promotion de l'élevage de bétail trypanotolérant en Afrique centrale et occidentale (RAF/88/100). Zimbabwe, Harare, Fao-Pnud, 211p.

8. FAO, 1994. Proposition d'une stratégie et d'un plan d'action pour le sous-secteur de l'élevage. Tcb/Ben/2353 (A). Rome, Italie, Fao, 197 p.

9. FEO, 1994. Rapport annuel d'activité (M dr/D e/Pdpa). O kpara, Bénin, $\mathrm{Feo}, 50 \mathrm{p}$.

10. FEO, 1995. Rapport annuel d'activité (Mdr/D e/Pdpa). O kpara, Bénin, Feo, $62 \mathrm{p}$.

11. FEO, 1996. Rapport annuel d'activité (Mdr/De/Pdpa). O kpara, Bénin, Feo, $56 \mathrm{p}$.
12. FEO, 1997. Rapport annuel d'activité (Mdr/De/Pdpa). O kpara, Bénin, Feo, $65 \mathrm{p}$.

13. HOSTE C., CLOE L., DESLAN DES P., POIVEY J.P., 1983. Etude de la production laitière et de la croissance des veaux de vaches allaitantes N'Dama et Baoulé en Côte d'Ivoire. II. Relations entre la production laitière et la croissance des veaux. Revue Elev. Méd. vét. Pays trop. 36 : 207-213

14. NJOYA A., BOUCHEL D., NGO TAMA A.C., PLANCHENAULT D. 1998. Facteurs affectant le poids à la naissance, la croissance et la viabilité des veaux en milieu paysan au nord du Cameroun. Revue Elev. M éd. vét. Pays trop., 51 : 335-343.

15. PLANCHENAULT D., TRAORE M.T., ROY F., TALL S.H., 1986. Amélioration génétique des bovins $N$ 'Dama. II. Croissance des veaux avant sevrage au ranch de Madina-Diassa, Mali. Revue Elev. Méd. vét. Pays trop., 39 : 51-57.

16. POIVEY J.P., MENISSIER F., VISSAC B., MOUSSA K., 1987. Variabilité de la croissance des veaux et jeunes bovins dans les troupeaux sédentaires du nord de la Côte d'Ivoire. Revue Elev. M éd. vét. Pays trop., $40: 157-166$

17. SAN O USSI I., 1993. Proposition sur la réorientation des activités de la Ferme Elevage de l'O kpara. O kpara, Bénin, Pdpa, 4 p.

18. SAS, 1989. SAS/STAT U ser's guide, vers. 6, 4th ed. Cary, NC, USA, SAS Inst.

19. SEARLE R., 1971. Linear models. New York, London, Sydney, Toronto, John Wiley and Sons, $531 \mathrm{p}$.

20. SINSIN B., OLOUTANT S., AHANCHEDE A., OUMOROU A. 1989. Etudes agrostologiques et bromatologiques dans le périmètre de Nikki, Kalalé et Ségbana. Rapport final. Abomey Calavi, Bénin, Fsa/U nb, $120 \mathrm{p}$.

21. YOUSSAO A.K.I., 1998. Amélioration génétique de la race bovine Borgou. Etude de quelques facteurs non-génétiques influençant le poids et la croissance des veaux à la Ferme de I'O kpara au Bénin. Mémoire DES en Sciences vétérinaires tropicales, U niversité de Liège, Belgique, 73 p.

Reçu le 06.03.2000, accepté le 01.02.2001 


\section{Summary}

Youssao A.K.I., Ahissou A., Michaux C., Farnir F., Touré Z. Idrissou N.-D., Leroy P.L. Nongenetic factors influencing the weight and growth of Borgu calves at the Okpara Breeding Farm in Benin

A study was carried out from records on 453 calves kept at the O kpara Breeding Farm between 1994 and 1998. Nongenetic factors affecting the birth weight, weight at three and twelve months of age as well as average daily gains from birth to three months, birth to twelve months and three to twelve months were investigated. A linear model comprising the fixed effects of birth season, birth year, dam age at calving and sex was used to perform the analysis of variance by the least squares. The birth season had a significant effect $(p<0.001)$ on weight and average daily gains. The best weight performance was observed in animals born during the rainy season. The birth year significantly $(p<0.001)$ influenced the birth weight, weight at twelve months and average daily gains from birth to twelve months. The effect of dam age at calving was observed in twelve-month-old calves $(p<0.05)$ and in average daily gains from birth to twelve months ( $<<0.05$ ). The male weight at birth was significantly higher than that of females $(p<0.05)$.

Key words: Borgu cattle - Calf - Birth weight - Weight gain Season - Age - M other - Sex - Growth factor - Benin.

\section{Resumen}

Youssao A.K.I., Ahissou A., Michaux C., Farnir F., Touré Z., Idrissou N.-D., Leroy P.L. Factores no genéticos que influencian el peso y el crecimiento de terneros de raza Borgu en la finca de cría de 0 kpara, Benin

De 1994 a 1998, se realizó, a partir de datos obtenidos con 453 terneros criados en la Finca de cría de 0 kpara, un estudio de algunos factores no genéticos que influencian el peso al nacimiento, a tres y a doce meses, así como las ganancias cotidianas promedio del nacimiento a tres meses, del nacimiento a doce meses y de tres a doce meses, en terneros Borgu. Se utilizó un modelo linear, incluyendo los efectos fijos de la época de nacimiento, el año de nacimiento, la edad de la madre al parto y del sexo, en el cuadro de un análisis de varianza. La época de nacimiento tuvo un efecto significativo $(p<0,001$ ) sobre los pesos y las ganancias cotidianas promedio. Los mejores rendimientos ponderales se obtuvieron con animales nacidos durante la época Iluviosa. El año de nacimiento tuvo una influencia significativa $(p<0,001)$ sobre el peso al nacimiento, a doce meses y la ganancia cotidiana media del nacimiento a doce meses. El efecto de la edad de la madre al parto fue observado en los terneros de doce meses $(p<0,05)$ y sobre la ganancia cotidiana media registrada del nacimiento a doce meses ( $p<0,05)$. Los machos presentaron un peso al nacimiento significativamente $(p<0,05)$ superior al de las hembras.

Palabras clave: Borgu ganado bovino - Ternero - Peso al nacimiento - Ganancia de peso - Estación - Edad - Madre - Sexo Factor de crecimiento - Benin. 